PROCEEDINGS OF THE

AMERICAN MATHEMATICAL SOCIETY

Volume 137, Number 10, October 2009, Pages 3379-3386

S 0002-9939(09)09903-1

Article electronically published on May 6, 2009

\title{
A MIXING-LIKE PROPERTY AND INEXISTENCE OF INVARIANT FOLIATIONS FOR MINIMAL DIFFEOMORPHISMS OF THE 2-TORUS
}

\author{
ALEJANDRO KOCSARD AND ANDRÉS KOROPECKI
}

(Communicated by Jane M. Hawkins)

\begin{abstract}
We consider diffeomorphisms in $\overline{\mathcal{O}}^{\infty}\left(\mathbb{T}^{2}\right)$, the $C^{\infty}$-closure of the conjugancy class of translations of $\mathbb{T}^{2}$. By a theorem of Fathi and Herman, a generic diffeomorphism in that space is minimal and uniquely ergodic. We define a new mixing-type property, which takes into account the "directions" of mixing, and we prove that generic elements of $\overline{\mathcal{O}}^{\infty}\left(\mathbb{T}^{2}\right)$ satisfy this property. As a consequence, we obtain a residual set of strictly ergodic diffeomorphisms without invariant foliations of any kind. We also obtain an analytic version of these results.
\end{abstract}

\section{INTRODUCTION}

In FH77, Fathi and Herman combined generic arguments with the so-called fast approximation by conjugations method of Anosov and Katok AK70 to study a particular class of diffeomorphisms of a compact manifold: the $C^{\infty}$-closure of the set of diffeomorphisms which are $C^{\infty}$ conjugate to elements of a locally free $\mathbb{T}^{1}$ action on the manifold. They proved that a generic element of that space is minimal and uniquely ergodic (i.e. there is a residual subset of such diffeomorphisms), in particular proving that every compact manifold admitting a locally free $\mathbb{T}^{1}$-action supports a minimal and uniquely ergodic diffeomorphism.

Surprisingly, the space studied by Fathi and Herman contains many elements with unexpected dynamical properties; for example, a generic diffeomorphism in that space is weak mixing [Her92, FS05, and if the underlying space is $\mathbb{T}^{n}$, the action of its derivative on the unit tangent bundle is minimal [Kor07. For a very complete survey on the technique of Anosov-Katok and its variations, see [FK04]. In [FS05, Fayad and Saprikyna use a real analytic version of this method to construct minimal weak mixing diffeomorphisms.

In this short article, we restrict our attention to diffeomorphisms of $\mathbb{T}^{2}$. In this setting, the closure of maps $C^{\infty}$ conjugated to elements of any locally free $\mathbb{T}^{1}$-action coincides with the $C^{\infty}$-closure of the conjugancy class of the rigid translations

$$
R_{\left(\lambda_{1}, \lambda_{2}\right)}:(x, y) \mapsto\left(x+\lambda_{1}, y+\lambda_{2}\right),
$$

Received by the editors March 26, 2008, and, in revised form, January 25, 2009.

2000 Mathematics Subject Classification. Primary 37E30, 37B05.

The authors were supported by CNPq-Brazil.

(C)2009 American Mathematical Society Reverts to public domain 28 years from publication 
that is, the set $\overline{\mathcal{O}}^{\infty}\left(\mathbb{T}^{2}\right)$, where

$$
\mathcal{O}\left(\mathbb{T}^{2}\right)=\left\{h R_{\alpha} h^{-1}: h \in \operatorname{Diff}{ }^{\infty}\left(\mathbb{T}^{2}\right), \alpha \in \mathbb{T}^{2}\right\}
$$

As we mentioned above, a generic element of $\overline{\mathcal{O}}^{\infty}\left(\mathbb{T}^{2}\right)$ is topologically weak mixing; however, no topologically mixing elements are known. It is also unknown if a minimal diffeomorphism of $\mathbb{T}^{2}$ can be topologically mixing. In fact, no examples of minimal $C^{\infty}$ diffeomorphisms of $\mathbb{T}^{2}$ in the homotopy class of the identity are known other than the ones in $\overline{\mathcal{O}}^{\infty}\left(\mathbb{T}^{2}\right)$.

Recall that a homeomorphism $f: \mathbb{T}^{2} \rightarrow \mathbb{T}^{2}$ is topologically weak mixing if $f \times f$ is transitive. An equivalent definition is the following: for each open $U \subset \mathbb{T}^{2}$ and $\epsilon>0$, there is $n>0$ such that $f^{n}(U)$ is $\epsilon$-dense in $\mathbb{T}^{2}$. We will be interested in a similar property, which implies weak mixing but is stronger in that it requires open sets to be mixed in every homological direction.

Definition 1.1. A homeomorphism $f: \mathbb{T}^{2} \rightarrow \mathbb{T}^{2}$ is weak spreading if for a lift $\hat{f}: \mathbb{R}^{2} \rightarrow \mathbb{R}^{2}$ of $f$ the following holds: for each open set $U \in \mathbb{R}^{2}, \epsilon>0$ and $N>0$, there is $n>0$ such that $\hat{f}^{n}(U)$ is $\epsilon$-dense in a ball of radius $N$.

Let $\overline{\mathcal{O}}_{\mu}^{\infty}\left(\mathbb{T}^{2}\right)$ denote the area-preserving elements of $\overline{\mathcal{O}}^{\infty}\left(\mathbb{T}^{2}\right)$. Now we can state our main theorem.

Theorem 1.2. Weak spreading diffeomorphisms are generic in $\overline{\mathcal{O}}^{\infty}\left(\mathbb{T}^{2}\right)$ and $\overline{\mathcal{O}}_{\mu}^{\infty}\left(\mathbb{T}^{2}\right)$.

As a consequence, we prove a result about invariant foliations announced by Herman in FH77] without proof. By a topological foliation we mean a codimension1 foliation of class $C^{0}$, that is, a partition $\mathcal{F}$ of $\mathbb{T}^{2}$ into one-dimensional topological submanifolds which is locally homeomorphic to the partition of the unit square by horizontal segments. We say that the foliation is invariant by $f$ if $f(F) \in \mathcal{F}$ for every $F \in \mathcal{F}$. We then have:

Corollary 1.3. The set of diffeomorphisms in $\overline{\mathcal{O}}^{\infty}\left(\mathbb{T}^{2}\right)$ (resp. $\overline{\mathcal{O}}_{\mu}^{\infty}\left(\mathbb{T}^{2}\right)$ ) without any invariant topological foliation is residual in $\overline{\mathcal{O}}^{\infty}\left(\mathbb{T}^{2}\right)$ (resp. $\overline{\mathcal{O}}_{\mu}^{\infty}\left(\mathbb{T}^{2}\right)$ ).

Since the set of minimal and uniquely ergodic diffeomorphisms in $\overline{\mathcal{O}}^{\infty}\left(\mathbb{T}^{2}\right)$ (or $\overline{\mathcal{O}}_{\mu}^{\infty}\left(\mathbb{T}^{2}\right)$ ) is also residual, this provides a residual set of minimal, uniquely ergodic diffeomorphisms with no invariant foliations.

Using the ideas of [FS05], it is possible to construct real analytic examples, working with diffeomorphisms which have an analytic extension to a band of fixed width in $\mathbb{C}^{2}$ (see the precise definitions in $\$ 5$ ).

Theorem 1.4. The set of real analytic diffeomorphisms of $\mathbb{T}^{2}$ which are weak spreading is residual in $\overline{\mathcal{O}}_{\rho}^{\omega}\left(\mathbb{T}^{2}\right)$.

Remark 1.5. We use the word "weak" in the definition of weak spreading because there is an analogy with the topological weak mixing property. We could also define strong spreading (or just spreading) as the property that for any open set $U \subset \mathbb{R}^{2}$, $\epsilon>0$ and $N>0$ there is $n_{0}$ such that $\hat{f}^{n}(U)$ is $\epsilon$-dense in a ball of radius $N$ whenever $n>n_{0}$. This would be in analogy with the definition of topological mixing, but it is clearly a stronger property. In fact, the typical examples of topologically mixing systems in $\mathbb{T}^{2}$ mix only in one direction (e.g. Anosov systems and timeone maps of some minimal flows Fay02). It is not obvious that strong spreading 
diffeomorphisms exist; however, as P. Boyland kindly explained to us, an example of a strong spreading diffeomorphism can be constructed using Markov partitions and the techniques of Boy08.

\section{The Method of FAthi-Herman}

As usual, we identify $\mathbb{T}^{2} \simeq \mathbb{R}^{2} / \mathbb{Z}^{2}$ with the quotient projection $\pi: \mathbb{R}^{2} \rightarrow \mathbb{T}^{2}$, and we denote by $\operatorname{Diff}^{\infty}\left(\mathbb{T}^{2}\right)$ the space of $C^{\infty}$ diffeomorphisms of $\mathbb{T}^{2}$. A lift of one such diffeomorphism $f$ to $\mathbb{R}^{2}$ is a map $\hat{f}: \mathbb{R}^{2} \rightarrow \mathbb{R}^{2}$ such that $f \pi=\pi \hat{f}$. If $f$ is homotopic to the identity, this is equivalent to saying that $\hat{f}$ commutes with integer translations, i.e. $\hat{f}(z+v)=\hat{f}(z)+v$ for $v \in \mathbb{Z}^{2}$. Two different lifts of a diffeomorphism of $\mathbb{T}^{2}$ always differ by a constant $v \in \mathbb{Z}^{2}$. We will denote by $\hat{R}_{\alpha}$ the translation $z \mapsto z+\alpha$ of $\mathbb{R}^{2}$ and by $R_{\alpha}$ the rotation of $\mathbb{T}^{2}$ lifted by $\hat{R}_{\alpha}$.

The method used in [FH77], adapted to our case, can be resumed as follows:

Lemma 2.1. Let $P \subset \overline{\mathcal{O}}^{\infty}\left(\mathbb{T}^{2}\right)\left(\right.$ or $\left.\overline{\mathcal{O}}_{\mu}^{\infty}\left(\mathbb{T}^{2}\right)\right)$ be such that

(1) $P=\bigcap_{n \geq 0} P_{n}$, where the $P_{n}$ are open;

(2) For each $g \in \operatorname{Diff}^{\infty}\left(\mathbb{T}^{2}\right)$ (resp. Diff ${ }_{\mu}^{\infty}\left(\mathbb{T}^{2}\right)$ ) and $m \in \mathbb{N}$, there is $N>0$ such that $\left\{g f g^{-1}: f \in P_{n}\right\} \subset P_{m}$ whenever $n>N$;

(3) For each $n \in \mathbb{N}, p / q \in \mathbb{Q}$, there exists $h \in \operatorname{Diff}^{\infty}\left(\mathbb{T}^{2}\right)$ (resp. Diff ${ }_{\mu}^{\infty}\left(\mathbb{T}^{2}\right)$ ) such that

- $h R_{(1 / q, 0)}=R_{(1 / q, 0)} h$,

- $h R_{\alpha_{k}} h^{-1} \in P_{n}$ for some sequence $\alpha_{k} \rightarrow(p / q, 0)$.

Then, $P$ is residual in $\overline{\mathcal{O}}^{\infty}\left(\mathbb{T}^{2}\right)$ (resp. $\overline{\mathcal{O}}_{\mu}^{\infty}\left(\mathbb{T}^{2}\right)$ ).

Proof. Given $m \in \mathbb{N}, p / q \in \mathbb{Q}$, and $g \in \operatorname{Diff}^{\infty}\left(\mathbb{T}^{2}\right)$, let $n$ be as in (2), and then let $h$ and $\left\{\alpha_{k}\right\}$ be as in (3). Then

$$
P_{n} \ni h R_{\alpha_{k}} h^{-1} \underset{k \rightarrow \infty}{\stackrel{C^{\infty}}{\longrightarrow}} h R_{(p / q, 0)} h^{-1}=R_{(p / q, 0)},
$$

so that

$$
P_{m} \ni g\left(h R_{\alpha_{k}} h^{-1}\right) g^{-1} \underset{k \rightarrow \infty}{\stackrel{C^{\infty}}{\longrightarrow}} g R_{(p / q, 0)} g^{-1} .
$$

This proves that $g R_{(p / q, 0)} g^{-1} \in \bar{P}_{m}^{\infty}$. Since this holds for all $g$ and $p / q$, it follows that $P_{m}$ is dense in $\overline{\mathcal{O}}^{\infty}\left(\mathbb{T}^{2}\right)$ because so is the set

$$
\left\{h R_{(p / q, 0)} h^{-1}: h \in \operatorname{Diff}^{\infty}\left(\mathbb{T}^{2}\right), p / q \in \mathbb{Q}\right\} .
$$

Since this holds for all $m$ and each $P_{m}$ is open, this proves that $P$ is residual in $\overline{\mathcal{O}}^{\infty}\left(\mathbb{T}^{2}\right)$. The proof in the area-preserving case is the same.

The property of having no invariant topological foliations is hard to deal with in the $C^{\infty}$ topology in order to apply the above lemma. However, the weak spreading property can be adequately described as an intersection of countably many properties that fit well into the lemma; thus we first prove Theorem 1.2 using the above method, and then we prove that weak spreading is not compatible with the existence of invariant foliations of any kind, which implies Corollary 1.3 . 


\section{Proof of Theorem 1.2}

Let $P_{n}$ denote the set of all $f \in \overline{\mathcal{O}}^{\infty}\left(\mathbb{T}^{2}\right)$ such that if $\hat{f}$ is a lift of $f$, for any ball $B$ of radius $1 / n$ in $\mathbb{R}^{2}$, there is $k>0$ such that $\hat{f}^{k}(B)$ is $1 / n$-dense in a ball of radius $n$. Note that if this property holds for some lift, it holds for any lift of $f$.

It is clear that $P=\bigcap P_{n}$ is the set of weak spreading elements of $\overline{\mathcal{O}}^{\infty}\left(\mathbb{T}^{2}\right)$. Denote by $B(z, \epsilon)$ the ball of radius $\epsilon$ centered at $z$. Given a lift $\hat{f}$ of some $f \in P_{n}$, and $z \in \mathbb{R}^{2}$, let $k_{z}$ be the smallest positive integer such that $\hat{f}^{k_{z}}(B(z, 1 / n))$ is $1 / n$-dense in a ball of radius $n$. By continuity of $\hat{f}$, the map $z \mapsto k_{z}$ is upper semi-continuous, and therefore it attains a maximum $K$ when $z \in[0,1]^{2}$. But since $\hat{f}$ lifts a map homotopic to the identity, $k_{z}=k_{z+v}$ when $v \in \mathbb{Z}^{2}$, so that $k_{z} \leq K$ for all $z \in \mathbb{R}^{2}$. Hence, if $g$ is close enough to $f$ in the $C^{0}$ topology and $\hat{g}$ is the lift of $g$ closest to $\hat{f}$, it also holds that $\hat{g}^{k_{z}}(B(z, 1 / n))$ is dense in a ball of radius $n$ for any $z \in \mathbb{R}^{2}$. Hence $P_{n}$ is open in the $C^{0}$ topology (and, in particular, in the $C^{\infty}$ topology).

To see that condition (2) of Lemma 2.1 holds, note that any lift $\hat{g}$ of a diffeomorphism $g$ of $\mathbb{T}^{2}$ is bi-Lipschitz. Fix $m \in \mathbb{N}$, let $C$ be a Lipschitz constant for $\hat{g}$ and $\hat{g}^{-1}$, and let $n>0$ be such that $C<n / m$. If $\hat{f}$ is a lift of $f \in P_{n}$, and if

$U$ is an open set, then there is $k$ such that $\hat{f}^{k}\left(\hat{g}^{-1}(U)\right)$ is $1 / n$-dense in a ball of radius $n$. Thus, $\hat{g} \hat{f}^{k} \hat{g}^{-1}(U)$ is $C / n$-dense in a ball of radius $n / C$, which implies that $g f g^{-1} \in P_{m}$, as required.

To finish the proof, it remains to see that condition (3) of Lemma 2.1 holds. To do this, it suffices to construct, for each $q, n \in \mathbb{N}$, a diffeomorphism $h \in \operatorname{Diff}^{\infty}\left(\mathbb{T}^{2}\right)$ which commutes with $R_{(1 / q, 0)}$ and such that $h R_{(\alpha, 0)} h^{-1} \in P_{n}$ whenever $\alpha$ is irrational. Note that it is enough to prove this for some multiple of $q$ instead of $q$. We will assume that $q$ is a multiple of $n$ and $q \geq 2 n$, since otherwise we may use $2 q n$ instead of $q$. We define $h$ by constructing a lift $\hat{h}=\hat{v} \circ \hat{u}$, where $\hat{v}, \hat{u}: \mathbb{R}^{2} \rightarrow \mathbb{R}^{2}$ are the maps

$$
\hat{u}(x, y)=(x, y+m \cos (2 \pi q x)), \quad \hat{v}(x, y)=(x+n \cos (2 \pi q y), y)
$$

and $m$ is a sufficiently large integer that we will choose later. It is clear that $\hat{u}$ and $\hat{v}$ are lifts of $C^{\infty}$ torus diffeomorphisms in the homotopy class of the identity, because they commute with integer translations. They also commute with $\hat{R}_{(1 / q, 0)}$ and $\hat{R}_{(0,1 / q)}$ as well. The same properties hold for $\hat{h}=\hat{v} \circ \hat{u}$. Moreover, since both $\hat{u}$ and $\hat{v}$ are area-preserving, so is $\hat{h}$, and the rest of the proof also works in the area-preserving setting.

Let $\delta=2 n(\pi q m)^{-1}, I_{\delta}=[-\delta, \delta] \times\{0\}$, and $J_{\delta}=\left[(4 q)^{-1}-\delta / 2,(4 q)^{-1}+\delta / 2\right] \times\{0\}$.

Claim 1. If $m$ is large enough, then $\hat{h}\left(I_{\delta}\right)$ is contained in the ball of radius $1 /(2 n)$ centered at $(n, m)$, and $\hat{h}\left(J_{\delta}\right)$ is $1 / n$-dense in $[-n, n] \times[-n, n]$.

Proof. First observe that from the inequality

$$
1-\cos (x) \leq x^{2} / 2 \quad \forall x \in \mathbb{R},
$$

it follows that (denoting by $\left(x_{1}, x_{2}\right)_{i}$ the coordinate $x_{i}$ )

$$
\left|(\hat{u}(x, 0)-\hat{u}(0,0))_{2}\right| \leq 2 m(\pi q x)^{2}<2 m(\pi q \delta)^{2}=8 n^{2} / m
$$

if $|x|<\delta$. Since $\hat{u}(0,0)=(0, m)$, this means that $\hat{u}\left(I_{\delta}\right)$ is contained in the rectangle $[-\delta, \delta] \times[m-b, m+b]$, where $b=8 n^{2} / m$. By the definition of $\hat{v}$ and a similar 


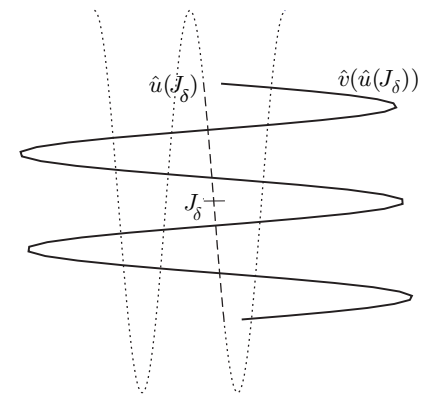

FiguRE 1. Image of $J_{\delta}$ by $\hat{h}$

argument (since $m$ is an integer), we can conclude that $\hat{v}\left(\hat{u}\left(I_{\delta}\right)\right) \subset[n-a, n+a] \times$ $[m-b, m+b]$, where

$$
a=\delta+2 n(\pi q b)^{2}=2 n(\pi q m)^{-1}+128 \pi^{2} q^{2} n^{5} m^{-2} .
$$

Since both $a$ and $b$ can be made arbitrarily small if $m$ is large enough, $\hat{h}\left(I_{\delta}\right)$ is contained in a ball around $(n, m)$ of radius $1 /(2 n)$ if $m$ is large enough.

For the second part of the claim, note that

$$
\cos (x+\pi / 2)=\sin (x) \geq x / 2 \quad \text { if } 0 \leq x \leq \pi / 2
$$

so that

$$
\left(\hat{u}\left((4 q)^{-1}+\delta / 2,0\right)-\hat{u}\left((4 q)^{-1}, 0\right)\right)_{2}=m \cos (\pi q \delta+\pi / 2) \geq m \pi q \delta / 2=n,
$$

and similarly

$$
\left(\hat{u}\left((4 q)^{-1}-\delta / 2,0\right)-\hat{u}\left((4 q)^{-1}, 0\right)\right)_{2}=-n .
$$

Thus $\hat{u}\left(J_{\delta}\right)$ is an arc that transverses the rectangle $[-\delta / 2, \delta / 2] \times[-n, n]$ vertically.

Let $L=\{0\} \times[-n, n]$. Note that $\hat{v}(L)$ is $1 / q$-dense in $[-n, n] \times[-n, n]$, since every rectangle of the form $[-n, n] \times[-n+k / q,-n+(k+1) / q], 0 \leq k \leq 2 q n-1$ is horizontally transversed by $\hat{v}(L)$. By the previous paragraph, $\hat{u}\left(J_{\delta}\right)$ contains a point of the form $(s, y)$ with $|s|<\delta / 2$ for each $(0, y) \in L$. Since $\hat{v}(s, y)=\hat{v}(0, y)+(s, 0)$, it follows from the previous facts that, if $m$ is so large that $\delta / 2<1 / q$, then $\hat{h}\left(J_{\delta}\right)=$ $\hat{v}\left(\hat{u}\left(J_{\delta}\right)\right)$ is $2 / q$-dense in $[-n, n] \times[-n, n]$ (see Figure 1 ). Since we assumed earlier that $q \geq 2 n$, we conclude that $h\left(J_{\delta}\right)$ is $1 / n$-dense in $[-n, n] \times[-n, n]$, as claimed. This proves the claim.

Let $B \subset \mathbb{R}^{2}$ be a ball of radius $1 / n$. Then $B$ contains a ball $B^{\prime}$ of radius $1 /(2 n)$ around some point with coordinates $(i / q, j / q)$, where $i, j$ are integers (because $q \geq 2 n)$. Since $\hat{h}$ commutes with $R_{(1 / q, 0)}$, and using Claim 1 , we see that

$$
\hat{h}\left(I_{\delta}+(i / q, j / q)-(n, m)\right)=\hat{h}\left(I_{\delta}\right)-(n, m)+(i / q, j / q) \subset B^{\prime} .
$$

In particular, $I_{\delta}+(i / q, j / q)-(n, m) \subset \hat{h}^{-1}(B)$. Since $J_{\delta}$ lies on the same horizontal line as $I_{\delta}$ and is shorter than $I_{\delta}$, if $\alpha$ is an irrational number, we can find $k \in \mathbb{N}$ and $r \in \mathbb{Z}$ such that $J_{\delta}+(r, 0) \subset \hat{R}_{(\alpha, 0)}^{k}\left(I_{\delta}\right)$, and we have

$$
J_{\delta}+(i / q, j / q)-(n, m)+(r, 0) \subset \hat{R}_{(\alpha, 0)}^{k}\left(I_{\delta}+(i / q, j / q)-(n, m)\right) .
$$


Thus, if $\hat{f}=\hat{h} \hat{R}_{(\alpha, 0)} \hat{h}^{-1}$, then

$$
\begin{aligned}
& \hat{f}^{k}(B)=\hat{h} \hat{R}_{(\alpha, 0)}^{k} \hat{h}^{-1}(B) \supset \hat{h} \hat{R}_{(\alpha, 0)}^{k}\left(I_{\delta}+(i / q, j / q)-(n, m)\right) \\
& \supset \hat{h}\left(J_{\delta}+(i / q, j / q)-(n+r, m)\right)=\hat{h}\left(J_{\delta}\right)+(i / q, j / q)-(n+r, m),
\end{aligned}
$$

which is just a translation of $\hat{h}\left(J_{\delta}\right)$, and thus by Claim 1 it is $1 / n$-dense in some ball of radius $n$. That is, $\hat{f}^{k}(B)$ is $1 / n$-dense in some ball of radius $n$, which means that $h R_{(\alpha, 0)} h^{-1} \in P_{n}$. Since $\alpha$ was an arbitrary irrational number, this completes the proof.

\section{INVARIANT FOLIATIONS}

Corollary 1.3 is a direct consequence of Theorem 1.2 and the next two propositions.

Proposition 4.1. If $\mathcal{F}$ is a foliation of $\mathbb{T}^{2}$ and $\hat{\mathcal{F}}$ is the lift of $\mathcal{F}$ to $\mathbb{R}^{2}$, then there is a leaf $F \in \hat{\mathcal{F}}$ which is contained in a strip bounded by two parallel straight lines $L$ and $L^{\prime}$ such that both lines belong to different connected components of $\mathbb{R}^{2}-F$.

Proof. If $\mathcal{F}$ has a compact leaf, then there is $z \in \mathbb{R}^{2}$ and a leaf $F$ of $\hat{\mathcal{F}}$ such that $F+(p, q)=F$, for some pair of integers $(p, q) \neq(0,0)$. Thus, assuming $p \neq 0$, if $L_{0}$ is a line of slope $q / p$, it follows that $s=\sup \left\{d\left(z, L_{0}\right): z \in F\right\}<\infty$, and the proposition follows by choosing $L$ and $L^{\prime}$ a distance greater than $s$ apart from $L_{0}$, one on each side. If $p=0$, then $q \neq 0$ and an analogous argument holds.

Now suppose $\mathcal{F}$ has no compact leaves. By [HH83, Theorem 4.3.3], $\mathcal{F}$ is equivalent to a foliation $\mathcal{F}^{\prime}$ obtained by suspension of the trivial foliation $\mathbb{R} \times \mathbb{T}^{1}$ over an orientation-preserving circle homeomorphism $f: \mathbb{T}^{1} \rightarrow \mathbb{T}^{1}$ with irrational rotation number. Such a foliation has a lift $\hat{\mathcal{F}}^{\prime}$ to $\mathbb{R}^{2}$ such that the intersection of the leaf through $(0, y)$ with the line $\{n\} \times \mathbb{R}$ is at $\left(n, \hat{f}^{n}(y)\right)$, where $\hat{f}: \mathbb{R} \rightarrow \mathbb{R}$ is a lift of $f$. If $\phi(y)$ denotes the length of the arc of leaf joining $(0, y)$ to $(1, \hat{f}(y))$, then $\phi: \mathbb{R} \rightarrow \mathbb{R}$ is a continuous function and it is $\mathbb{Z}$-periodic, because $\hat{\mathcal{F}}^{\prime}$ is a lift of a foliation of $\mathbb{T}^{2}$. Thus there is a constant $C$ such that $\phi(x)<C$ for all $x \in \mathbb{R}$. Note that the length of the arc joining $(n, y)$ to $(n+1, \hat{f}(y))$ is also bounded by $C$.

If $\rho$ is the rotation number of $\hat{f}$, by classic results for circle homeomorphisms (see, for example, [MvS93]) we have $\left|\hat{f}^{n}(y)-y-n \rho\right| \leq 1$ for all $n \in \mathbb{Z}$ and $y \in \mathbb{R}$. Let $F^{\prime}$ be a leaf of $\hat{\mathcal{F}}^{\prime}$ containing the point $(0, y)$. Then $F^{\prime}=\bigcup_{n \in \mathbb{Z}} F_{n}^{\prime}$, where $F_{n}$ is the arc joining $\left(n, \hat{f}^{n}(y)\right)$ to $\left(n+1, \hat{f}^{n+1}(y)\right)$. Note that the distance from $\left(n, \hat{f}^{n}(y)\right)$ to the line $L_{0}$ of slope $\rho$ through $(0, y)$ is at most 1 , and the length of $F_{n}^{\prime}$ is at most $C$. Thus the distance from any point of $F^{\prime}$ to $L_{0}$ is at most $C+1$.

We know that $\mathcal{F}$ is equivalent to $\mathcal{F}^{\prime}$, which means there is a homeomorphism $h: \mathbb{T}^{2} \rightarrow \mathbb{T}^{2}$ mapping leaves of $\mathcal{F}^{\prime}$ to leaves of $\mathcal{F}$. If $\hat{h}: \mathbb{R}^{2} \rightarrow \mathbb{R}^{2}$ is a lift of $h$, then we can write $\hat{h}(z)=A(z)+\psi(z)$, where $A \in \mathrm{GL}(2, \mathbb{Z})$ and $\psi$ is a $\mathbb{Z}^{2}$-periodic function, bounded by some constant $K$. If $L_{1}=A L_{0}, z=\hat{h}\left(z^{\prime}\right)$ is a point in $F=h\left(F^{\prime}\right)$, and $w=A\left(w^{\prime}\right)$ is a point in $L_{1}$, then

$$
|z-w|=\left|A\left(z^{\prime}-w^{\prime}\right)+\psi(z)\right| \leq\|A\| \cdot\left|z^{\prime}-w^{\prime}\right|+K \leq\|A\|(C+1)+K,
$$

the last inequality following from the fact that $z^{\prime} \in F^{\prime}$ and $w^{\prime} \in L_{0}$. It follows that $s=\sup _{z \in F} d\left(z, L_{1}\right)<\infty$, and as before we complete the proof by choosing $L$ and $L^{\prime}$ parallel to $L_{1}$ and a distance at least $s$ apart from $L_{1}$, one on each side. 
Proposition 4.2. If $f$ is weak spreading and homotopic to the identity, then $f$ has no invariant topological foliations.

Proof. By Proposition 4.1, if $\mathcal{F}$ is a foliation invariant by $f$ and $\hat{\mathcal{F}}$ is the lift of this foliation to $\mathbb{R}^{2}$ (hence invariant by $\hat{f}$ ), then there is a leaf $\hat{F}_{0} \in \hat{\mathcal{F}}$ which is contained in a strip bounded by two parallel lines $L$ and $L^{\prime}$ and which contains each of those lines in a different component of its complement. Let $u$ be a unit vector orthogonal to $L$. We will assume without loss of generality that $u$ has a nonzero second coordinate. If $S$ is the strip bounded by $\hat{F}_{0}$ and $\hat{F}_{0}+(0,1)$, denoting by $\phi_{u}: \mathbb{R}^{2} \rightarrow \mathbb{R}$ the orthogonal projection onto the direction of $u$, it is clear that

$$
\operatorname{width}_{u}(S) \doteq \operatorname{diam}\left(\phi_{u}(S)\right)<\infty .
$$

Moreover, $\bigcup_{n \in \mathbb{Z}} S+(0, n)=\mathbb{R}^{2}$.

For each $n \in \mathbb{Z}$, since $\hat{f}^{n}\left(\hat{F}_{0}\right)$ cannot cross $\hat{F}_{0}+(0, k)$ for any $k \in \mathbb{Z}$, we see that $\hat{f}^{n}\left(\hat{F}_{0}\right) \subset S+(0, m)$ for some $m \in \mathbb{Z}$. This implies that $\operatorname{width}_{u}\left(f^{n}\left(\hat{F}_{0}\right)\right) \leq M=$ $\operatorname{width}_{u}(S)$. But then

$$
\hat{f}^{n}\left(\hat{F}_{0}+(0,1)\right) \subset S+(0, m+1)
$$

so that $\hat{f}^{n}(S)$ is contained in the strip bounded by $\hat{F}_{0}+(0, m)$ and $\hat{F}_{0}+(0, m+$ $2)$. This means that $\operatorname{width}_{u}\left(\hat{f}^{n}(S)\right) \leq 2 M$. However, if $f$ is weak spreading, then there is $n>0$ such that $\hat{f}^{n}(S)$ is $1 / 3$-dense in some ball of radius $3 M$, so that $\operatorname{width}_{u}\left(\hat{f}^{n}(S)\right)>2 M$, contradicting the previous claim. This completes the proof.

\section{The real analytic Case}

In this section we briefly explain how to obtain minimal weak spreading analytic diffeomorphisms of $\mathbb{T}^{2}$. We kindly thank the anonymous referee for bringing this to our attention.

First we introduce some notation, following [FS05]. Fix $\rho>0$, and let $g: \mathbb{R}^{2} \rightarrow$ $\mathbb{R}^{2}$ be any real analytic $\mathbb{Z}^{2}$-periodic function which can be holomorphically extended to $A_{\rho}=\left\{(z, w) \in \mathbb{C}^{2}:|\operatorname{Im} z|<\rho,|\operatorname{Im} w|<\rho\right\}$. We define $\|g\|_{\rho}=\sup _{A_{\rho}}|g(z, w)|$, and we denote by $C_{\rho}^{\omega}\left(\mathbb{T}^{2}\right)$ the space of all functions of this kind which satisfy $\|g\|_{\rho}<\infty$.

Let Diff ${ }_{\rho}^{\omega}\left(\mathbb{T}^{2}\right)$ be the space of all diffeomorphisms $f$ of $\mathbb{T}^{2}$ which are homotopic to the identity and which have a lift whose periodic part is in $C_{\rho}^{\omega}\left(\mathbb{T}^{2}\right)$. There is a metric in $\operatorname{Diff}_{\rho}^{\omega}\left(\mathbb{T}^{2}\right)$ defined by

$$
d_{\rho}(h, k)=\inf _{(p, q) \in \mathbb{Z}^{2}}\|\hat{h}-\hat{k}+(p, q)\|_{\rho}
$$

where $\hat{h}$ and $\hat{k}$ are lifts of $h$ and $k$, respectively. Since $C_{\rho}^{\omega}\left(\mathbb{T}^{2}\right)$ is a Banach space, it is easy to see that the metric $d_{\rho}$ turns $\operatorname{Diff}{ }_{\rho}^{\omega}\left(\mathbb{T}^{2}\right)$ into a complete metric space.

To apply the arguments of the previous sections we work in the space $\overline{\mathcal{O}}_{\rho}^{\omega}\left(\mathbb{T}^{2}\right)$ defined as the closure in the $d_{\rho}$ metric of the set of diffeomorphisms of the form $h R_{\alpha} h^{-1}$, where $\alpha \in \mathbb{T}^{1}$, and $h \in \operatorname{Diff}_{\rho}^{\omega}\left(\mathbb{T}^{2}\right)$ is any diffeomorphism whose lifts to $\mathbb{R}^{2}$ have a bi-holomorphic extension to $\mathbb{C}^{2}$.

We observe that the proof of Lemma 2.1 applies to this setting if we use $\overline{\mathcal{O}}_{\rho}^{\omega}\left(\mathbb{T}^{2}\right)$ instead of $\overline{\mathcal{O}}^{\infty}\left(\mathbb{T}^{2}\right)$ (and the topology induced by $d_{\rho}$ instead of the $C^{\infty}$ topology). 
To complete the proof of Theorem 1.4, we note that everything in $\S 3$ works without any modifications, because the function $h$ constructed to obtain property (3) of Lemma 2.1 has an analytic extension to all of $\mathbb{C}^{2}$ which is a bi-holomorphism.

\section{ACKNOWLEDGMENTS}

We are grateful to E. Pujals and P. Boyland for useful discussions and to the anonymous referee for bringing the results of [FS05] to our attention and for suggesting various improvements, in particular the content of \$5.

\section{REFERENCES}

[AK70] D. Anosov and A. Katok, New examples in smooth ergodic theory. Ergodic diffeomorphisms, Transactions of the Moscow Mathematical Society 23 (1970), 1-35. MR0370662 (51:6888)

[Boy08] P. Boyland, Transitivity of surface dynamics lifted to abelian covers, preprint, 2008.

[FH77] A. Fathi and M. Herman, Existence de difféomorphismes minimaux, Astérisque 49 (1977), 37-59. MR0482843(58:2889)

[Fay02] B. Fayad, Weak mixing for reparameterized linear flows on the torus, Ergodic Theory \& Dynamical Systems 22 (2002), 187-201. MR1889570 (2003a:37012)

[FK04] B. Fayad and A. Katok, Constructions in elliptic dynamics, Ergodic Theory \& Dynamical Systems 24 (2004), 1477-1520. MR2104594 (2008b:37031)

[FS05] B. Fayad and M. Saprykina, Weak mixing disc and annulus diffeomorphisms with arbitrary Liouville rotation number on the boundary, Ann. Sci. École Norm. Sup. (4) 38 (2005), no. 3, 339-364. MR2166337 (2006g:37001)

[HH83] G. Hector and U. Hirsch, Introduction to the geometry of foliations, Part A: Fundamentals, Friedr. Vieweg \& Sohn, 1983. MR881799 (88a:57048)

[Her92] M. R. Herman, On the dynamics of Lagrangian tori invariant by symplectic diffeomorphisms, Progress in Variational Methods in Hamiltonian Systems and Elliptic Equations (L'Aquila, 1990), Longman Science and Technology, Harlow, 1992 (Pitman Research Notes Mathematical Series, 243), pp. 92-112. MR.1176346 (93h:58054)

[Kor07] A. Koropecki, On the dynamics of torus homeomorphisms, Ph.D. thesis, IMPA, 2007.

[dMvS93] W. de Melo and S. van Strien, One-dimensional dynamics, Ergebnisse der Mathematik und ihrer Grenzgebiete (3) [Results in Mathematics and Related Areas (3)], vol. 25, Springer-Verlag, Berlin, 1993. MR.1239171 (95a:58035)

Instituto de Matemática, Universidade Federal Fluminense, Rua Mário Santos Braga S/N, 24020-140 Niteroi, RJ, BraziL

E-mail address: alejo@impa.br

Instituto de Matemática, Universidade Federal Fluminense, Rua Mário Santos Braga S/N, 24020-140 Niteroi, RJ, BRAZIL

E-mail address: koro@mat.uff.br 